\title{
Screening for Hydroxychloroquine Retinal Toxicity: Is It Necessary?
}

\author{
C. D. G. MORSMAN, ${ }^{1}$ S. J. LIVESEY,${ }^{1}$ I. M. RICHARDS,${ }^{2}$ J. D. JESSOP ${ }^{2}$ \\ P. V. MILLS ${ }^{1}$ \\ Cardiff
}

\begin{abstract}
Summary
All the patients (73) in the Rheumatology Department five year study of second line therapy who have taken hydroxychloroquine (Plaquenil) for rheumatoid arthritis for longer than 18 months were reviewed. These patients have their treatment dosage carefully monitored and have been receiving regular ophthalmic examinations. Most patients still taking the drug were assessed with a battery of tests for evidence of retinal toxicity. No retinal toxicity causing visual loss was found.

On the basis of these results and a review of the recent literature we no longer routinely screen patients for hydroxychloroquine retinal toxicity in Cardiff.
\end{abstract}

Hydroxychloroquine has been shown to be an effective agent in the treatment of rheumatoid arthritis and other connective tissue diseases. ${ }^{1}$ There have been numerous reports of visual loss caused by chloroquine ${ }^{2}$ and it has been assumed that this effect would be similar in its analogue, hydroxychloroquine. Reports of classical 4 aminoquinolone maculopathy affecting vision as a result of hydroxychloroquine therapy are hard to find in the literature $^{3,4}$ and Mackenzie ${ }^{5}$ suggests that retinal toxicity does not occur at a daily dose below $6.5 \mathrm{mg} / \mathrm{kg}$ lean body weight. Other authors ${ }^{6,7,8,9,10}$ confirm a low incidence (Table I).

Most authors still recommend ophthalmo-

Table I Incidence of toxicity-previous publications

\begin{tabular}{|c|c|c|}
\hline Mackenzie $^{5}$ & 1983 & 0 in 900 \\
\hline Mikkelsen $^{6}$ & 1979 & 0 in 338 \\
\hline Rynes $^{7}$ & 1983 & 4 in 99 \\
\hline Finbloom et al. ${ }^{8}$ & 1985 & 0 in 66 \\
\hline${\text { Mäntyjärvi }{ }^{9}}^{9}$ & 1985 & 1 in 63 \\
\hline Johnson \& Vine ${ }^{10}$ & 1987 & 0 in 8 \\
\hline
\end{tabular}

logical examination at six to twelve monthly intervals, ${ }^{5,7,8,10,11}$ and the manufacturers suggest two to four checks per year, specifying fundoscopy and central field testing with a red target. ${ }^{12}$ It has been suggested that rheumatologists are capable of carrying out the screening, ${ }^{13}$ but many rheumatologists are less happy than the Edinburgh group at performing fundoscopy and an alternative, easier and more reliable test would be required.

The criteria to justify any screening programme include; the presence of an important health problem, a benefit in early treatment, a suitable test for detecting the problem and a benefit which outweighs the adverse effects ${ }^{14}$ (Table II).

In view of this background our prospective

Table II Criteria for screening ${ }^{\text {lt }}$

1. important health problem

2. benefit of early treatment

3. suitable test

4. benefit outweighs adverse effects

Departments of Ophthalmology ${ }^{1}$ and Rheumatology ${ }^{2}$, University Hospital of Wales, Cardiff.

Correspondence to: Mr D Morsman, FCOphth, Dept of Ophthalmology, University Hospital of Wales, Heath Park, Cardiff CF4 3RH. 
Table III. Distribution of study patients

\begin{tabular}{ll}
\hline Baseline & $\begin{array}{l}\text { Notes lost } \\
\text { Systemic side effects/ineffective/died }\end{array}$ \\
No baseline & 63 \\
63 & 76
\end{tabular}

study was set up in 1980 to see if routine ophthalmological screening helps to prevent visual loss in patients with rheumatoid arthritis treated with hydroxychloroquine at recommended dosage.

\section{Patients and Methods}

The records of all patients enrolled in the Department of Rheumatology five year study of second line therapy and randomly assigned to treatment with hydroxychloroquine were examined where possible. These patients suffered from rheumatoid arthritis and received hydroxychloroquine as their main medication apart from non steroidal anti-inflammatory drugs. Patients with significant hepatic or renal dysfunction or other contraindication to hydroxychloroquine therapy were excluded. Standard dosage was $400 \mathrm{mg}$ per day in two divided doses, though in one of our cases this was reduced to $200 \mathrm{mg}$ because of prolonged remission.

The patients were assessed in the eye clinic within the first 12 weeks of treatment and at approximately annual intervals thereafter. The standard assessment included Snellen visual acuity, and fundoscopy following pupillary dilation. Patients who were still taking the drug in 1989 were assessed with additional tests as follows: colour vision (Ishihara pseudoisochromatic plates, Amsler grid (red and white), macular threshold and central $10^{\circ}$ field to a red stimulus (Freidmann Mk2 analyser) at $0.6 \mathrm{log}$ units above foveal threshold.

\section{Results}

One hundred and sixty-one patients were seen in the Eye Clinic. Seventy-two discontinued therapy within 18 months because of lack of effect or less commonly systemic side effects. Four patients died for reasons unrelated to their rheumatoid disease or treatment and later records were unavailable for a further five (Table V).
Of all patients seen within the first three months of therapy, 36 of 151 had a macular pigmentary disturbance $(23 \%)$ though in only ten patients was this thought to be severe enough to prejudice our ability to screen for toxicity. The average age was significantly different in these two groups being 57 for those without, and 64 for those with pigmentation. This would suggest that the appearance of abnormal macular pigmentation is more likely to be related to ageing than to drug toxicity. Interestingly three of these ten patients continued therapy despite this finding without any deterioration in vision over periods of 2 to 3 years.

Information was available for 73 patients ( 52 female and 21 male) who continued treatment for longer than 18 months (maximum seven years). Thirty-one patients discontinued therapy prior to 1989 and were assessed on the basis of visual acuity and fundoscopy (Table IV, group A).

Two patients developed macular abnormalities and treatment was stopped as a result (Table V). One of them (Table VI, case 1) weighed only $30 \mathrm{~kg}$, though she received $400 \mathrm{mg}$ hydroxychloroquine per day (about twice the dose recommended by Mackenzie). Visual acuity was $6 / 9$ and the patient was symptom free. There was no further deterioration over the subsequent 12 months and this may represent a toxic maculopathy though there were no features to distinguish it from age related changes. The other patient (Table VI, case 2) received an appropriate dose for her weight, but pre-existing drusen appeared to become more prominent with degredation of the foveal reflex. Acuity was unchanged at

Table IV. Patients at risk of retinopathy

Continued therapy $>18$ months 73

Stopped HCQ prior to 1989 (Group A) 31

Still on HCQ (Group B) 
Table V. Results of examinations

\begin{tabular}{ll}
\hline Group A & 31 patients \\
\hline $\begin{array}{l}\text { HCQ discontinued } \\
\text { Acuity Fundoscopy }\end{array}$ & $\begin{array}{l}2(6 \%) \text { pigmentary maculopathy } \\
\text { (Cases 1 and 2) }\end{array}$ \\
\hline Group B & 42 patients \\
\hline Still on HCQ & 9 pigment changes \\
Acuity Ishihara & 2 Amsler only \\
Amsler grid Friedmann & 3 Friedmann only \\
Fundoscopy & 1 Amsler \& Friedmann (Case 3) \\
\hline
\end{tabular}

6/6 and fluorescein angiography failed to show any abnormality other than the drusen. Clearly these changes were age related rather than toxic, though treatment was stopped because of possible difficulties with screening. Four other patients with normal vision developed minor pigmentary changes at the maculae which were not present at the time of the baseline check. All these patients were over the age of 63 and the changes were not felt to be related to therapy or severe enough to interfere with screening.

Forty-two patients underwent the full assessment detailed above (Table IV, group B). Two noted blurring of areas of the Amsler grid without a field defect on the Freidmann analyser. One of these patients had significant cataract reducing vision to $6 / 12$. Three patients had defects on the Friedmann field testing. Two of these defects were inconsistent on repeated testing and the other was a $0.8 \mathrm{log}$ unit defect at a single point in each eye (not homonymous) in the presence of posterior subcapsular lens opacities. This did not alter over a further six months of treatment. Percival ${ }^{15}$ found $6 \%$ of controls to be unreliable at central field testing (albeit by a different technique), and these three patients probably represent a similar group.

One patient had a defect in one eye on both Amsler grid and Freidmann field analysis (Table VI case 3). This was an inferior arcuate distribution without any abnormality of retina or optic disc, or any symptoms. On balance this was not thought to be related to drug ingestion, though treatment was discontinued for safety. No significant change occurred in the subsequent nine months. The patient's weight was $56 \mathrm{~kg}$ (this is below the $61.5 \mathrm{~kg}$ where the standard dosage should be reduced according to Mackenzie's figures).
Nine patients developed minor pigmentary changes at the maculae though vision was $6 / 9$ or better in all cases. One patient had several microaneuryms in one retina (no cause was found).

Colour vision testing was unhelpful. One case of congenital colour blindness was confirmed.

In summary, only three patients had their treatment discontinued because of ophthalmological screening. Two received an excessive dose according to their weight and the other did not have any evidence of drug toxicity.

\section{Discussion}

If our cases are added to those quoted in Table I, over 1500 patients have been monitored and only five possible cases of retinal toxicity found. Mackenzie's ${ }^{5}$ cases were followed for a mean of seven years and no toxicity was found at a dose of $6.5 \mathrm{mg} / \mathrm{kg} / \mathrm{day}$. Mikkelsen ${ }^{6}$ titrated dose against corneal changes to avoid retinal toxicity. Rynes ${ }^{7}$ admits that his screening procedures tend to overdiagnose toxicity; two of his patients resumed treatment without further problem, one had a minor reversible field defect in one eye only, and the fourth had symptoms more suggestive of migraine than retinal toxicity. Finbloom et al. ${ }^{8}$ found toxicity with chloroquine but not hydroxychloroquine. Mäntyjärvi's ${ }^{9}$ single case of possible

Table VI. Cases of possible toxicity

\begin{tabular}{lrcc}
\hline Case & 1 & 2 & 3 \\
\hline weight $(\mathrm{kg})$ & 30 & 64 & 52 \\
total dose $(\mathrm{g})$ & 300 & 219 & 510 \\
mg/kg/day & 13 & 6.25 & 7.7 \\
acuity & $6 / 9$ & $6 / 6$ & $6 / 6$ \\
age & 65 & 63 & 52 \\
\hline
\end{tabular}


toxicity acquired a defect of colour vision only (this is not thought to be a reliable test for hydroxychloroquine toxicity ${ }^{4,15}$ ) and Johnson and Vines ${ }^{10}$ patients received between one and $4 \mathrm{~kg}$ without toxicity.

4-aminoquinolone retinal toxicity presents initially as a premaculopathy involving asymptomatic paracentral scotomata and macular pigmentary disturbance which is reversible on cessation of treatment. Later changes involve more extensive permanent damage to the central retina with visual loss (progressing to a 'bull's.eye' maculopathy). ${ }^{11}$ With respect to hydroxychloroquine, this later maculopathy has only been described a few times in patients receiving high doses. ${ }^{3,4}$

Numerous tests have been proposed for screening, and the variety suggests lack of an ideal.

(1) Fundoscopy: Scherbel et al. showed a high incidence of pigmentary maculopathy indistinguishable from toxicity in patients with rheumatoid arthritis in $1965 .^{16}$

(2) Field: Central field loss seems the most logical defect to pursue. Percival showed tangent screen testing to be unhelpful; $6 \%$ of controls had defects and $40 \%$ of patients had abnormal red central fields without other evidence of maculopathy. ${ }^{15}$ Static perimetry on an instrument such as the Friedmann analyser is probably more effective. ${ }^{17}$ The Amsler grid is a quick, easy test which can be done in a rheumatology clinic or even by patients in their own homes. Easterbrook ${ }^{18}$ and others $^{19,20}$ have found it sensitive and if a screening test is needed this is probably the best currently available.

(3) Colour vision testing has generally been found to be unhelpful. ${ }^{4,15}$

(4) Contrast sensitivity: Fleck et al. ${ }^{13}$ found no abnormal results though Bishara ${ }^{21}$ found $44 \%$ in patients taking hydroxychloroquine!

(5) Electrodiagnostic tests are abnormal in a high proportion of patients receiving 4 aminoquinolones, ${ }^{22,23}$ but may be normal in the presence of maculopathy. ${ }^{4}$ This probably represents a reversible side effect of treatment rather than true toxicity.

Initially screening was based on fundoscopy as outlined in a previous publication. ${ }^{24} \mathrm{Sub}$ sequent analysis of our results (above) suggests that this is unreliable. Pigmentary granularity developed in $20 \%$ of cases but this was not associated with measurable loss of visual function. This appearance is increasingly common with advancing age in the absence of hydroxychloroquine therapy, and will produce a significant number of false positive results, as will most other tests in isolation.

We would suggest that our screening criteria (Table II) are not met.

(1) At appropriate dosage toxicity is rare or possibly absent.

(2) Early treatment (withdrawal of drug) would be beneficial only if retinal toxicity occurs and could be diagnosed accurately.

(3) Easterbrook has found the Amsler grid reliable and we would accept this subject to limitations induced by distortion in the optical media.

(4) The adverse effect concerning withdrawal of hydroxychloroquine is a flare up of the disease, or toxicity from alternative therapy such as gold or penicillamine. ${ }^{25}$

Both these possibilities present more risk to the patient than the very small one of retinal toxicity. Changing treatment on the basis of a false-positive result of an unreliable test could be extremely unfortunate.

As a result of this and the other recent studies (Table I) the Department of Ophthalmology in Cardiff no longer carries out regular examinations of patients taking hydroxychloroquine. A baseline examination involving visual acuity, Amsler grid and fundoscopy is done and the rheumatologist is notified of the findings. He may reasonably choose to continue treatment despite the presence of ocular disease if he considers that the potential benefit outweighs the risks. The effect of hydroxychloroquine in the presence of age related maculopathy is not well documented though our three patients did well. This initial examination may well be superfluous but provides a defence if coincidental ocular pathology is later ascribed to hydroxychloroquine therapy by the patient.

Most rheumatologists claim to monitor their patients according to the requirements 
of the manufacturer's data sheet. ${ }^{26}$ This puts an unnecessary workload on their ophthalmological colleagues by virtue of the very specific schedule quoted. Patients can be protected more effectively by using a dosage not exceeding $6.5 \mathrm{mg} / \mathrm{kg} / \mathrm{day}$, and checking renal and hepatic function periodically. Examining the patient's medical records is more useful than examining their eyes.

\section{References}

${ }^{1}$ Adams EM, Yocum DE, Bell CL: Hydroxychloroquine in the treatment of rheumatoid arthritis. Am J Med 1983, 75: 321-6.

${ }^{2}$ Marks JS: Chloroquine retinopathy; is there a safe daily dose? Ann Rheum Dis 1982, 41: 52-8.

${ }^{3}$ Shearer RV, Dubois EL: Ocular changes induced by long term hydroxychloroquine therapy. Am J Ophthalmol 1967, 64: 245-52.

${ }^{4}$ Adlakha D, Crews SJ, Shearer ACI, Tonks EL: Electrodiagnosis in drug induced disorders of the eye. Trans Ophthalmol Soc UK 1967, 89: 267-84.

${ }^{5}$ Mackenzie AH: Dose refinements in long term therapy of rheumatoid arthritis with antimalarials. Am J Med 1983, (Supp): 40-45.

${ }^{6}$ Mikkelsen J: Ocular complications of treatment with antimalarial drugs in dermatology. Ugeskr Laeg 1979, 141: 2316-18.

${ }^{7}$ Rynes RI: Ophthalmologic safety of long term hydroxychloroquine sulfate treatment. $\mathrm{Am} \mathrm{J} \mathrm{Med}$ 1983, Supp: 35-39.

${ }^{8}$ Finbloom DS, Silver K, Newsome DA, Gunkel R: Comparison of Hydroxychloroquine and chloroquine use and the development of retinal toxicity. J Rheumatol 1985; 12: 692-4.

${ }^{9}$ Mantyjarvi M: Hydroxychloroquine treatment and the eye. Scand J Rheumatol 1985; 14: 171-4.

${ }^{10}$ Johnson MW, Vine AK: Hydroxychloroquine therapy in massive doses without retinal toxicity. $\mathrm{Am} \mathrm{J}$ Ophthalmol 1987, 104: 139-44.

${ }^{11}$ Bernstein HN: Ophthalmologic considerations and testing in patients receiving long term antimalarial therapy. Am J Med 1983, Supp: 25-34.
${ }^{12}$ Walker G: ABPI Data Sheet compendium 1988-89; London, Datapharm, 1989; 1516.

${ }^{13}$ Fleck BW, Bell AL, Mitchell JD, Thompson BJ, Hurst NP, Nuki G: Screening for antimalarial maculopathy in rheumatology clinics. $\mathrm{Br}$ Med $\mathrm{J}$ 1985, 291: 782-5.

${ }^{14}$ Khaw KT: Screening: the need for a balance. Eye 1989, 3: (editorial).

${ }^{15}$ Percival SPB and Meanock I: Chloroquine: ophthalmological safety and clinical assessment in rheumatoid arthritis. $\mathrm{Br}$ Med J 1968, 3: 579-84.

${ }^{16}$ Scherbel AL, Mackenzie AH, Nousek JE, Atdjian M: Ocular lesions in rheumatoid arthritis and related disorders with particular reference to retinopathy. $N$ Engl J Med 1965, 273: 360-6.

${ }^{17}$ Friedmann AI: The early detection of chloroquine retinopathy with the Friedmann visual field analyser. Ophthalmologica 1969, 158 (supp): 583-91.

${ }^{18}$ Easterbrook M: The sensitivity of Amsler grid testing in early chloroquine retinopathy. Trans $\mathrm{Oph}$ thalmol Soc UK 1985, 104: 204-7.

${ }^{19}$ Frenkel M: Safety of hydroxychloroquine (letter). Arch Ophthalmol 1982, 100: 841.

${ }^{20}$ Spalton DJ: Chloroquine and the eye. $\mathrm{Br} \mathrm{J}$ Clin Pract supp 52: 50-55.

${ }^{21}$ Bishara SA and Matamoros N: Evaluation of several tests in screening for chloroquine maculopathy. Eye 1989, 3: 777-82.

${ }^{22}$ Sassaman FW, Cassidy JT, Alpern M, Maaseidvaag F: Electro-retinography in patients with connective tissue disease treated with hydroxychloroquine. Am J Ophthalmol 1970, 70: 515-23.

${ }^{23}$ Arden GB and Kolb H: Antimalarial therapy and early retinal changes in patients wtih rheumatoid arthritis. Br Med J 1966, 1: 270-83.

${ }^{24}$ Mills PV, Beck M, Power BJ: Assessment of the retinal toxicity of hydroxychloroquine. Trans $O p h$ thalmol Soc UK 1981, 101: 109-13.

${ }^{25}$ Husain Z. and Runge LA: Treatment complications of rheumatoid arthritis with gold, hydroxychloroquine, D-penicillamine and levamisole. J Rheumatol 1980, 7: 825-30.

${ }^{26}$ Davis MJ and Dawes PT: Monitoring therapy in rheumatological practice. Br J Rheumatol 1990, 29 (Abst 1): 5 . 